\title{
Question and answer
}

Journal of Medical Genetics, 1980, 17, 57-58

\section{Question}

We read with interest the paper by Stewart and Stoll, ${ }^{1}$ which reported sibs with caudal regression who were born to a diabetic mother. We have just counselled a young diabetic woman who had given birth to a child with sacral agenesis and lateral facial dysplasia (hemifacial microsomia). When we examined the data from surveys reporting the association of diabetes with caudal regression, it appeared that congenital malformations, in particular caudal regression, were associated with diabetes of class $D$ and greater severity. The mothers of both our patient and the sibs reported by Stewart and Stoll were apparently class $C,{ }^{2}$ and from the reported surveys would not appear to be at significant risk for caudal regression. Is the concurrence of diabetes and caudal regression fortuitous in these patients, or have the reported surveys failed to detect a low level association? The latter hypothesis is perhaps not unexpected if caudal regression is considered to occur when genetic and environmental factors exceed a certain total or threshold. If the genetic susceptibility followed a 'normal' distribution, subjects at one extreme would have a marked genetic susceptibility and minimal environmental stress (for example, class $\mathrm{A}$, $\mathrm{B}$, or $\mathbf{C}$ diabetes) would result in the malformation. However, such subjects would be uncommon and could go undetected in large surveys. On the other hand, mothers with more severe diabetes $(\geqslant$ class D) provide a greater environmental stress and caudal regression might occur with a lower genetic susceptibility. The subjects with moderate or low genetic susceptibility are more common and therefore more likely to be detected in surveys. In addition there might well be a bias towards ascertainment of more severe classes of diabetes.

In any event we wonder if some of your more expert readers could comment upon this question.

Jane Evans ANd Alasdair Hunter

\section{References}

1 Stewart JM, Stoll S. Familial caudal regression anomalad and maternal diabetes. $J$ Med Genet 1979;16:17-20.

2 White P. Diabetes mellitus in pregnancy. Clin Perinatol $1974 ; 1: 331-47$.

\section{Answer}

Diabetes mellitus is a syndrome characterised by raised blood glucose levels. Several mechanisms are undoubtedly responsible, some of which may predispose to congenital malformations while others may not.

Since the offspring of diabetic fathers have not been noticed to have a higher incidence of congenital malformation, it is very probable that the abnormal metabolic environment of the mother is responsible for the increased malformation rate.

The incidence of all malformations is increased and among these is caudal regression, which although characteristic is extremely rare. For this reason patterns of association are difficult to establish.

There are a number of known facts which throw some light on the possible mechanisms. The incidence of congenital malformations is not increased in the offspring of gestational diabetic women. In these women, glucose intolerance returns to normal after the pregnancy and glucose intolerance is either absent or very mild in the first trimester of pregnancy. Provided that one assumes that this type of diabetic is not genetically distinct from the other forms, then this would suggest that the degree of metabolic abnormality is important.

Two other pieces of data would support this. The King's College Hospital Group have very preliminary data (presented to the British Diabetic Association) showing that diabetic women with very high $\mathrm{Hb} \mathrm{A_{1 }}$ levels in the first trimester produced malformed babies, while those with less abnormal values did not. This test provides a rough average of the mother's blood glucose over the life of the red blood cell: the higher the $\mathrm{Hb} \mathrm{A}_{1}$ the higher the blood glucose. Dr E M Deuchar has presented data to the 2nd Colloquium of Pregnancy and Diabetes $^{3}$ showing that rats made diabetic produced malformed fetuses. This could be reduced when the blood glucose levels of pregnant rats were returned towards normal with insulin treatment. The malformations she observed were various abnormalities of the vertebral column including caudal regression.

The classification of White ${ }^{2}$ is based on the age of onset, duration of diabetes, and the presence of complications, and does not take blood glucose levels into account after treatment. So why should class $\mathrm{D}$ upwards be more prone to caudal regression, 
and why should the case reported by Stewart and Stoll classified as class $C$ be affected?

Patients of class D upwards have had diabetes diagnosed before the age of 10 years, or have had the disease for more than $\mathbf{2 0}$ years, or have microangiopathic complications. In general, patients with very longstanding diabetes also have little or no endogenous insulin secretion and their diabetes is much more difficult to control. They tend to have much wider swings of blood glucose. It may well be the interaction of metabolic renal impairment, large and small vessel disease, together with indifferent diabetic control in the first trimester of pregnancy, which leads to the increased malformation rate. Any of these abnormalities when very marked may provide the sole mechanism.

It is of interest that in the case report of Stewart and Stoll ${ }^{\mathbf{1}}$ the mother received a single injection of $\mathrm{NPH}$, an intermediate-acting insulin. This would almost certainly lead to very poor control in half of any 24 hours. She received virtually no diabetic supervision and, by inference, control is likely to have been very poor.

There must also be interaction between the developing fetal tissue and the metabolic environ- ment since DM mothers will have one abnormal $\stackrel{\overparen{D}}{\stackrel{\leftrightarrow}{*}}$ fetus followed by a normal child. This is also sup- $\vec{\Rightarrow}$ ported by Dr Deuchar's study ${ }^{3}$ where only one to two fetuses of a litter were affected.

Finally, it is worth stating that it is estimated that 1 in 500 babies is born to a diabetic mother, so that $\frac{\vec{\sigma}}{8}$ in 10 years 60 babies will be born in Obstetric Units $\varrho$ delivering 3000 babies per year. Fewer than $5 \%$ के will have malformations incompatible with life $\overrightarrow{0}$ and one at most will have caudal regression. Analysis becomes very difficult unless data are pooled.

This is one of the reasons why a joint study has been set up between the Medical Services Study Group of the Royal College of Physicians, the Royal College of Obstetricians and Gynaecologists, and the British Diabetic Association. We intend to ir examine the malformation rate in the United Kingdom so that some of these questions can be answered with greater certainty.

\section{Reference}

${ }^{3}$ Deuchar EM. Proceedings of the 2nd Aberdeen International Colloquium on Carbohydrate Metabolism in Pregnancy and the Newborn (in press). 